\title{
Fiber-Optic Microsensors for Simultaneous Sensing of Oxygen and pH, and of Oxygen and Temperature
}

\author{
Anna S. Kocincova,t Sergey M. Borisov, ${ }^{\dagger}$ Christian Krause, ${ }^{\ddagger}$ and Otto S. Wolfbeis ${ }^{\star, t}$ \\ Institute of Analytical Chemistry, Chemo- and Biosensors, University of Regensburg, D-93040 Regensburg, Germany, and \\ Presens GmbH, Josef-Engert-Str. 11, D-93053 Regensburg, Germany
}

Fiber-optic microsensors with a tip diameter of $\sim 140 \mu \mathrm{m}$ have been developed that enable simultaneous measurement of dissolved oxygen (DO) and $\mathrm{pH}$, and of $\mathrm{DO}$ and temperature $(T)$, respectively. The tip of the optical fiber was covered with sensor compositions based on luminescent microbeads that respond to the respective parameters by a change in the decay time, intensity of their luminescence, or both. The use of microbeads enables the ratio of the signals to be easily varied, reduces the risk of fluorescence energy transfer between indicator dyes, and reduces the adverse effect of singlet oxygen that is produced in the oxygen-sensitive beads. The sensor chemistry for $\mathrm{DO} / \mathrm{pH}$ was modified

Oxygen, temperature, and $\mathrm{pH}$ are key parameters in environmental monitoring, ${ }^{1,2}$ marine research, ${ }^{3,4}$ the food industry, ${ }^{5-7}$ biotechnology, ${ }^{8-10}$ and medicine. ${ }^{11,12}$ Simultaneous measurement of two or more of these parameters is often required in complex media and processes.13,14 Moreover, knowing temperature is particularly important in optical sensing of oxygen since quenching by oxygen always is highly temperature dependent. ${ }^{15,16}$ Recently,

* Corresponding author. E-mail: otto.wolfbeis@chemie.uni-r.de. Tel.: (*49)(941) 943-4066. Fax: (*49) (941) 943-4064. Web: www.wolfbeis.de.

$\dagger$ University of Regensburg.

* Presens GmbH.

(1) Canete, F.; Rios, A.; Luque de Castro, M. D.; Valcarcel, M. Analyst 1987, 112, 263-266.

(2) Preininger, C.; Klimant, I.; Wolfbeis, O. S. Anal. Chem. 1994, 66, 18411846.

(3) Fernández-Valdivielso, C.; Egozkue, E.; Matías, I. R.; Arregui, F. G.; Bariáin, C. Sens. Actuators, B 2003, 91, 231-240.

(4) Zhao, Y.; Liao, Y. Sens. Actuators, B 2002, 86, 63-67.

(5) Marshall, A. J.; Blyth, J.; Davidson, C. A. B.; Lowe, C. R. Anal. Chem. 2003, 75, 4423-4431.

(6) Young, O. A.; Thomson, R. D.; Merhtens, V. G.; Loeffen, M. P. F. Meat Sci. 2004, 67 (1), 107-112.

(7) O’Mahony, F. C.; O’Riordan, T. C.; Papkovskaya, N.; Kerry, J. P.; Papkovsky, D. B. Food Control 2006, 17, 286-292.

(8) Deshpande, R. R.; Koch-Kirsch, Y.; Maas, R.; Krause, C.; Heinzle, E. Assay Drug Dev. Technol. 2005, 3, 299-307.

(9) Alderman, J. Biosens. Bioelectron. 2004, 19, 1529-1535.

(10) Zhang, Z.; Szita, N.; Boccazzi, P.; Sinskey, A. J.; Jensen, K. F. Biosens. Bioelectron. 2006, 93, 286-296.

(11) Mekhail, K.; Khacho, M.; Gunaratnam, L.; Lee, S. Cell Cycle 2004, 3, $1027-$ 1029.

(12) Stefanadis, C.; Tsiamis, E.; Vaina, S.; Toutouzas, K.; Boudoulas, H.; Gialafos, J.; Toutouzas, P. Am. J. Cardiol. 2004, 93, 207-210.

(13) Harms, P.; Kostov, Y.; French, J. A.; Soliman, M.; Anjanappa, M.; Ram, A.; Rao, G. Biotechnol. Bioeng. 2006, 93, 6-13.

(14) Borisov, S. M.; Krause, Ch.; Arain, S.; Wolfbeis, O. S. Adv. Mater. 2006, 18, 1511-1516.

(15) Coyle, L. M.; Gouterman, M. Sens. Actuators, B 1999, 61, 92-99. materials have been reported for simultaneous optical sensing of two parameters. ${ }^{17-20}$ Optical sensing is particularly advantageous over other methods since it makes possible virtually noninvasive measurements, e.g., through the glass window of a reaction vessel or bioreactor. In this case, a spot of sensing material is located on a wall inside the reaction vessel, while reading (by reflectance, fluorescence, etc.) is performed from the outside. There are, however, numerous situations where such a sensor spot cannot be easily located at the site to be monitored, for example, when it comes to performing measurements inside the body.

Microsensors represent an elegant solution in this limitation. Because of its size (20-200 $\mu \mathrm{m}$ are typical), a microsensor can be placed inside the body using a protective housing such as a steel needle and still will remain only minimally invasive. ${ }^{21}$ Fiberoptic microsensors are easier to fabricate than microelectrodes. While numerous kinds of fiber-optic chemical sensors and biosensors have been described so far, ${ }^{22}$ it must be noted that not a single fiber-optic (or other) sensor has survived on the market for in vivo monitioring of blood. One major reason (aside from problems associated with inflammation and sensor fouling) is the size of the sensor head, since at least three parameters are to be monitored in critical care medicine. If a single sensor tip can measure $n$ parameters simultaneously, the size required for the sensor array is reduced by a factor of $1 / n$, and the sample volume to a similar extent.

Microsensors capable of simultaneous measurement of several parameters also are the sensors of choice if high spatial resolution is required. Single microsensors have been reported for, for example, oxygen, ${ }^{23-25} \mathrm{pH},{ }^{26-28}$ carbon dioxide, ${ }^{29,30}$ or potassium

(16) Mongey, K. F.; Vos, J. G.; MacCraith, B. D.; McDonagh, C. M.; Coates, C.; McGarvey, J. J. J. Mater. Chem. 1997, 7, 1473-1479.

(17) Zelelow, B.; Khalil, G.; Phelan, G.; Carlson, B.; Gouterman, M.; Callis, J. B.; Dalton, L. R. Sens. Actuators, B 2003, 96, 304-314.

(18) Borisov, S. M.; Vasylevska, G. S.; Krause, C.; Wolfbeis, O. S. Adv. Funct. Mater. 2006, 16, 1536-1542.

(19) Vasylevska, A. S.; Borisov, S. M.; Krause, C.; Wolfbeis, O. S. Chem. Mater 2006, 18, 4609-4616.

(20) Koese, M. E.; Carrol, B. F.; Schanze, K. S. Langmuir 2005, 21, 91219129.

(21) Klimant, I.; Kühl, M.; Glud, R. N.; Holst, G. Sens. Actuators 1997, 38-39, 29-37.

(22) Wolfbeis, O. S. Anal. Chem. 2006, 78, 3859-3873; and previous biannual reviews.

(23) Rosenzweig, Z.; Kopelman, R. Anal. Chem. 1995, 67, 2650-2654.

(24) McNamara, K. P.; Li, X.; Stull, A. D.; Rosenzweig, Z. Anal. Chim. Acta 1998 , 361, 73-83.

(25) Park, E. J.; Reid, K. R.; Tang, W.; Kennedy, R. T.; Kopelman, R. L. Mater. Chem. 2005, 15, 2913-2919.

(26) Tan, W.; Shi, Z. Y.; Kopelman, R. Anal. Chem. 1992, 64, 2985-2990. 
Table 1. Materials Used for the Preparation of Sensor Cocktails SC-1 and SC-2 for Use in the Fiber-Optic Dual Microsensors

\begin{tabular}{|c|c|c|c|c|c|}
\hline \multirow[b]{2}{*}{ code } & \multirow[b]{2}{*}{ sensor for } & \multicolumn{3}{|c|}{ probes used to sense } & \multirow[b]{2}{*}{$\begin{array}{l}\text { polymer matrix (and } \\
\text { solvent for the polymer) }\end{array}$} \\
\hline & & oxygen & temperature & $\mathrm{pH}$ & \\
\hline SC-1 & $\mathrm{O}_{2}$ and $\mathrm{T}$ & $\begin{array}{l}\text { Pt-TFPP dissolved } \\
\text { in PS }\end{array}$ & $\begin{array}{l}\text { Ru-phen in PAN } \\
\text { microbeads }\end{array}$ & & PS (chloroform) \\
\hline SC-2 & $\mathrm{O}_{2}$ and $\mathrm{pH}$ & $\begin{array}{l}\text { Ru-dpp in ormosil } \\
\text { microbeads }\end{array}$ & & $\begin{array}{l}\text { HPTS in p-HEMA } \\
\text { microbeads }\end{array}$ & $\begin{array}{l}\text { polyurethane ( } 90 \% \\
\text { ethanol) }\end{array}$ \\
\hline
\end{tabular}

ion. ${ }^{31}$ To our knowledge, the only microsensor proposed so far that would enable simultaneous monitoring of two analytes was reported by Ji and Rosenzweig (a sensor for $\mathrm{Ca}^{2+}$ and $\mathrm{pH}$ ). ${ }^{32}$ It makes use of a mixture of two indicators, both immobilized at the tip of an optical fiber and excited with a laser beam. It requires measurement of fluorescence intensity (rather than decay time) at three wavelengths using a CCD camera. The approach made by Walt and co-workers, ${ }^{33,34}$ who positioned micrometer-sized sensing beads at the end of a fiber bundle array, is highly promising in terms of multianalyte sensing, but is intended for imaging purposes only and does not allow for dual sensing using a single fiber.

We report on two kinds of microsensors that are capable of simultaneous and continuous sensing of either $\mathrm{pO}_{2}$ (dissolved oxygen, DO) and temperature, or $\mathrm{pO}_{2}$ and $\mathrm{pH}$. A frequency domain method is applied for interrogation in order to warrant excellent long-term stability. The approach is fully compatible with a commercially available compact fiber-optic oxygen microsensor. ${ }^{35,36}$

\section{EXPERIMENTAL SECTION}

Materials. The temperature probe ruthenium(II) tris(1,10phenanthroline) dichloride hydrate (Ru-phen dichloride) and sodium 3-(trimethylsilyl)-1-propanesulfonate (Na-TMS) were from Aldrich (www.sigmaaldrich.com), platinum(II)-5,10,15,20-tetrakis(2,3,4,5,6-pentafluorphenyl)porphyrin (Pt-TFPP) was from Porphyrin Systems (www.porphyrin-systems.de), polyacrylonitrile (PAN; average MW 150 000) from Polysciences (www. polysciences.com), polystyrene (PS; 250 000) from Acros Organics (www.acros.com), and polyurethane hydrogel (type D4) from Cardiotech (www.cardiotech-inc.com).

The $\mathrm{pH}$-sensitive microbeads consisted of 8-hydroxypyrene1,3,6-trisulfonate (HPTS; from Fluka, www.sigmaaldrich.com) that was covalently immobilized (via its trisulfochloride) ${ }^{37}$ on an aminomodified poly(hydroxyethyl methacrylate) (p-HEMA) that was copolymerized with $\mathrm{N}$-aminoethylacrylamide) and was obtained from Presens ${ }^{38}$ (www.presens.de). The oxygen probe ruthenium(II)-tris-4,7-diphenyl-1,10-phenanthroline $\left[\mathrm{Ru}(\mathrm{dpp})_{3}(\mathrm{TMS})_{2}\right.$ salt] was synthesized as described before. ${ }^{39}$ The preparation of organically modified sol-gel beads ("ormosil") polymer was reported elsewhere, ${ }^{36}$ as was the preparation of the oxygen-sensitive ormosil beads (Ru-dpp in ormosil). ${ }^{19}$ The temperature-sensitive beads (Ruphen in PAN) were obtained as described before..$^{18}$ All chemicals and solvents were of analytical grade and used without further purification. Calibration gases (nitrogen and oxygen, each of 99.999\% purity) were purchased from Linde (www.linde-gase.de).

Doubly distilled water was used for the preparation of the buffer solutions. Their $\mathrm{pH}$ was controlled with a digital $\mathrm{pH}$ meter
(Knick, www.knick.de) calibrated at $20 \pm 1{ }^{\circ} \mathrm{C}$ with standard buffers of pH 7.0 and 4.0 (Merck; www.merck.de). The $\mathrm{pH}$ of solutions was adjusted (to within \pm 0.03 unit) to the desired value using MOPS buffers. These were adjusted to constant ionic strength $(I=140 \mathrm{mM})$ using sodium chloride as the background electrolyte. ${ }^{40}$

Preparation of the Microsensors. Table 1 gives an overview of the composition of the sensor materials ("cocktails") used in the work. Sensor cocktail SC-1 was prepared by first dissolving 1 $\mathrm{mg}$ of Pt-TFPP and $60 \mathrm{mg}$ of PS in $440 \mathrm{mg}$ of chloroform. Then, $14 \mathrm{mg}$ of Ru-phen/PAN microbeads was added, and the cocktail was stirred for $30 \mathrm{~min}$. The tip of a $140-\mu \mathrm{m}$ bifurcated optical glass fiber (from GP Fiber Optics; www.gp-fiberoptics.de) was coated with the respective sensor chemistry by immersing it into the respective cocktail and subsequently drying the material at ambient air for $24 \mathrm{~h}$ at room temperature. The material has an adhesion that is adequate for research purposes, but we assume that a treatment of the surface of the fiber tip with silanizing agents or a primer prior to coating with the sensor cocktail will probably improve adhesion and-hence-long-term stability.

Sensor cocktail SC-2 was prepared by dispersing $10 \mathrm{mg}$ of the Ru-dpp/ormosil microbeads and $14 \mathrm{mg}$ of the HPTS/p-HEMA microbeads in $500 \mathrm{mg}$ of a $5 \mathrm{wt} \%$ solution of the polyurethane hydrogel in an ethanol/water $(9: 1 ; \mathrm{v} / \mathrm{v})$ mixture. This cocktail was stirred overnight and then used for coating the optical fibers. Figure 1 shows photographic images of the microsensors of type SC-1 (left) and of type SC-2 (right) during excitation.

Components of the Optical System for Dual Sensing of $\mathbf{O}_{2} / \mathbf{T}$. The luminescence from the microsensors was read using a commercially available fiber-optic phase detection device. ${ }^{21}$ It is

(27) Kosch, U.; Klimant, I.; Wolfbeis, O. S. Fresenius J. Anal. Chem. 1999, 364, $48-53$.

(28) Song, A.; Parus, S.; Kopelman, R. Anal. Chem. 1997, 69, 863-867.

(29) Neurauter, G.; Klimant, I.; Wolfbeis, O. S. Fresenius J. Anal. Chem. 2000, $366,481-487$

(30) Ertekin, K.; Klimant, I.; Neurauter, G.; Wolfbeis, O. S. Talanta 2003, 59, 261-267.

(31) Werner, T.; Klimant, I.; Huber, C.; Krause, C.; Wolfbeis, O. S. Mikrochim. Acta 1999, 131, 25-28.

(32) Ji, J.; Rosenzweig, Z. Anal. Chim. Acta 1999, 397, 93-102.

(33) Ferguson, J. A.; Steemers, F. J.; Walt, D. R. Anal. Chem. 2000, 72, 56185624.

(34) Song, L.; Ahn, S.; Walt, D. R. Anal. Chem. 2006, 78, 1023-1033.

(35) Mock, T.; Dieckmann, G. S.; Haas, C.; Krell, A.; Tison, J. L.; Belem, A. L.; Papadimitriou, S.; Thomas, D. N. Aqut. Microb. Ecol. 2002, 29, 297-306.

(36) Klimant, I.; Ruckruh, F.; Liebsch, G.; Stangelmayer, A.; Wolfbeis, O. S. Microchim. Acta 1999, 131, 35-46.

(37) Offenbacher, H.; Wolfbeis, O. S.; Fuerlinger, E. Sens. Actuators 1986, 9, $73-84$.

(38) Klimant, I. Ger. Pat. Appl. 19829 657, 1997.

(39) Klimant, I.; Wolfbeis, O. S. Anal. Chem. 1995, 67, 3160-3166.

(40) Perrin, D. D.; Dempsey, B. Laboratory Manuals; Chapmann \& Hall: London, 1974. 


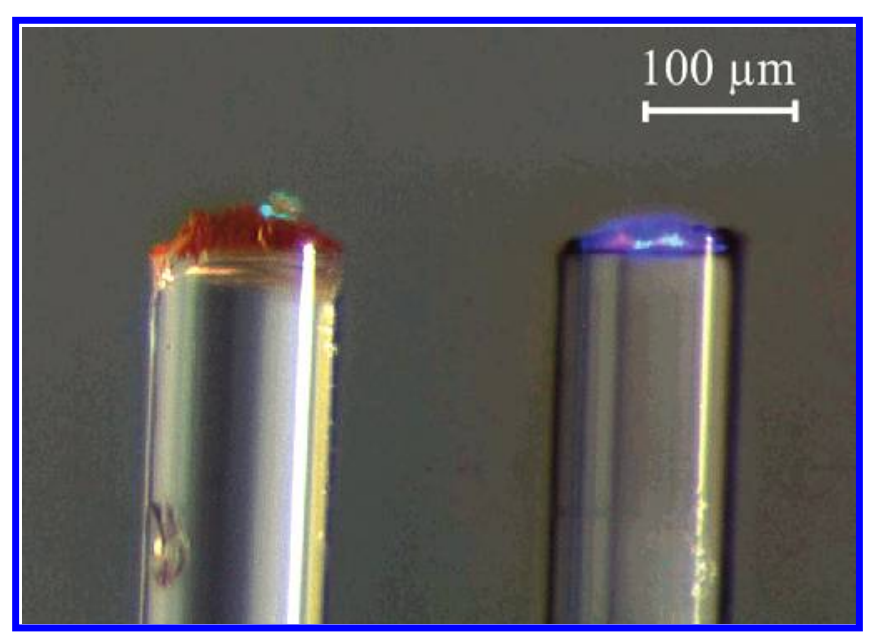

Figure 1. Photographic images of the microsensors of type SC-1 (left) and of type SC-2 (right) during excitation with a 505- and a 470$\mathrm{nm}$ LED, respectively. The left-side sensor tip $\left(\mathrm{O}_{2} / \mathrm{T}\right)$ displays a green color that results from the green $(505-\mathrm{nm})$ LED along with some red fluorescence resulting from the dual sensor. The right-hand sensor tip $\left(\mathrm{pH} / \mathrm{O}_{2}\right)$ displays the blue color of the 470-nm LED and a some red and green luminescence that results from the dual sensor.

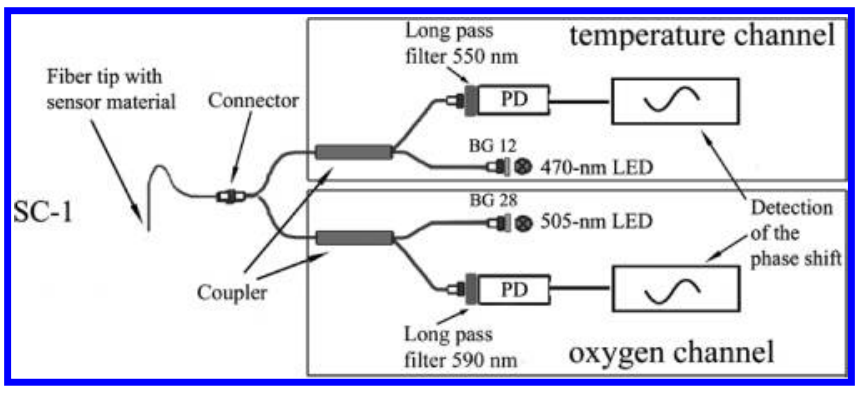

Figure 2. Components of the optical system used with the microsensors of type SC-1 $\left(\mathrm{O}_{2} / \mathrm{T}\right)$ using instruments Microx TX 3 and $\mathrm{pH}-1$ Micro devices.

schematically shown in Figure 2. In the case of applying sensor cocktail SC-1, the optical fiber was split into two fibers. One end was attached to the Microx TX 3 (the readout for the oxygen channel), the other to the $\mathrm{pH}-1$ Micro device (the temperature channel; both from Presens). This is schematically shown in Figure 2. Luminescence excitation was performed using the LEDs of the two reader devices. The light of 505-nm LED was filtered through a short-pass filter (BG 28; from Schott; www.schott.com), and the light from a 470-nm LED filtered through another shortpass filter (BG 12; Schott), respectively. Luminescence was detected by a photodiode (PD) after being filtered through a customized long-pass filter with a medium cutoff wavelength of $550 \mathrm{~nm}$ (pH-1 Micro) or a customized long-pass filter with a cutoff wavelength of $590 \mathrm{~nm}$ (Microx TX 3). The modulation frequencies were set to 60 and $5 \mathrm{kHz}$, respectively, for the $\mathrm{pH}-1$ Micro and the Microx system.
Components of the Optical System for Dual Sensing of $\mathrm{O}_{2} / \mathrm{pH}$. In the case of SC-2, the measurements were performed using a single pH-1 Micro device (see Figure 3). The excitation light of a 470-nm LED was modulated at two modulation frequencies of 30 and $60 \mathrm{kHz}$, respectively. Decay times were calculated using the equation $\tau=\tan \phi / 2 \pi f$, where $\phi$ is the measured phase shift and $f$ the modulation frequency of the respective LEDs.

Response Curve. Microsensors (10 of each type) were immersed into a $100-\mathrm{mL}$ glass beaker containing $30 \mathrm{~mL}$ of water (for SC-1) or buffer solution of defined pH (for SC-2). Nitrogen/ oxygen gas mixtures, whose composition and flow rate were controlled by a gas mixing device (GVS; from MKS; www.mksinst.com), were bubbled through the buffer solution to adjust the desired concentrations of dissolved oxygen. Temperature was controlled with a RC 6 cryostat (Lauda; www.lauda.de). All data for the sensor based on SC-2 ( $\mathrm{HH}$ and DO) were performed at $25{ }^{\circ} \mathrm{C}$.

Reproducibility tests and response curves were obtained by rapidly (within $1 \mathrm{~s}$ ) moving a microsensor from one beaker to another. The beakers contained sample solutions possessing different concentrations of the analytes (pH; oxygen) or temperatures as described in the Experimental Section.

Photographic images of the sensor cocktails spread as thin films (Figure 4) were acquired using a Leica DC 200 digital color camera mounted onto a Leica DMRE fluorescence microscope (www.leica-microsystems.com). The cocktails were coated on the polyester support to result in films after solvent evaporation. To avoid aggregation, the concentration of the beads in the films was reduced by a factor of 10 compared to the concentration in the microsensor. Photographic images of the sensor tips (Figure 1) were obtained using a megapixel CCD camera (Motic 1.3; type Moticam 1000; see www.motic.com) mounted onto a Zeiss stereomicroscope (type STEMI 2000-C; www.zeiss.com).

Experimental Data Fitting. 2D and 3D plots were fitted using Origin version 6.1 (www.originlab.com) and TableCurve 3D versus 3.12 (www.systat.com) software, respectively. The equations were solved using Maple software (vs 8; www.maplesoft.com).

\section{RESULTS AND DISCUSSION}

Choice of Materials. The principle of designing the materials capable of simultaneous sensing of two analytes is straightforward: two probes (e.g., an oxygen-sensitive and a temperaturesensitive probe) are designed and then combined so that crosssensitivity to the other analyte is avoided or minimized. That is best achieved by using microbeads that can be dispersed in a single polymer matrix that needs to be permeable, however, to both parameters (in this case $\mathrm{DO} / \mathrm{T}$ or $\mathrm{DO} / \mathrm{pH}$ ). For sensor materials such as for $\mathrm{pO}_{2} / \mathrm{pH}$ (SC-2), this approach also allows for some fine-tuning of the response range of a sensor by varying the ratio of the quantity of beads.

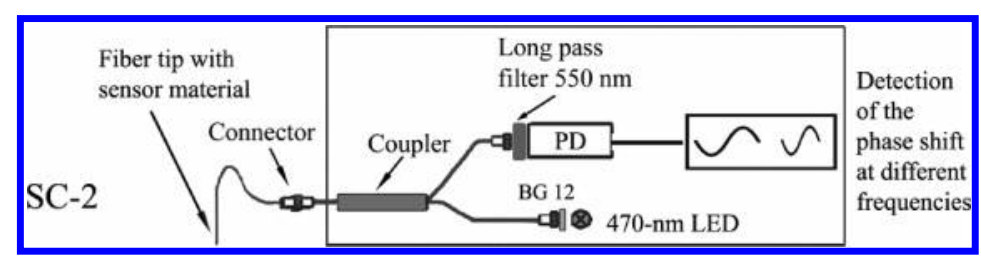

Figure 3. Components of the optical system used with the microsensors of type SC-2 $\left(\mathrm{pH} / \mathrm{O}_{2}\right)$ using a phase-resolving reader ( $\left.\mathrm{pH}-1 \mathrm{Micro}\right)$. 


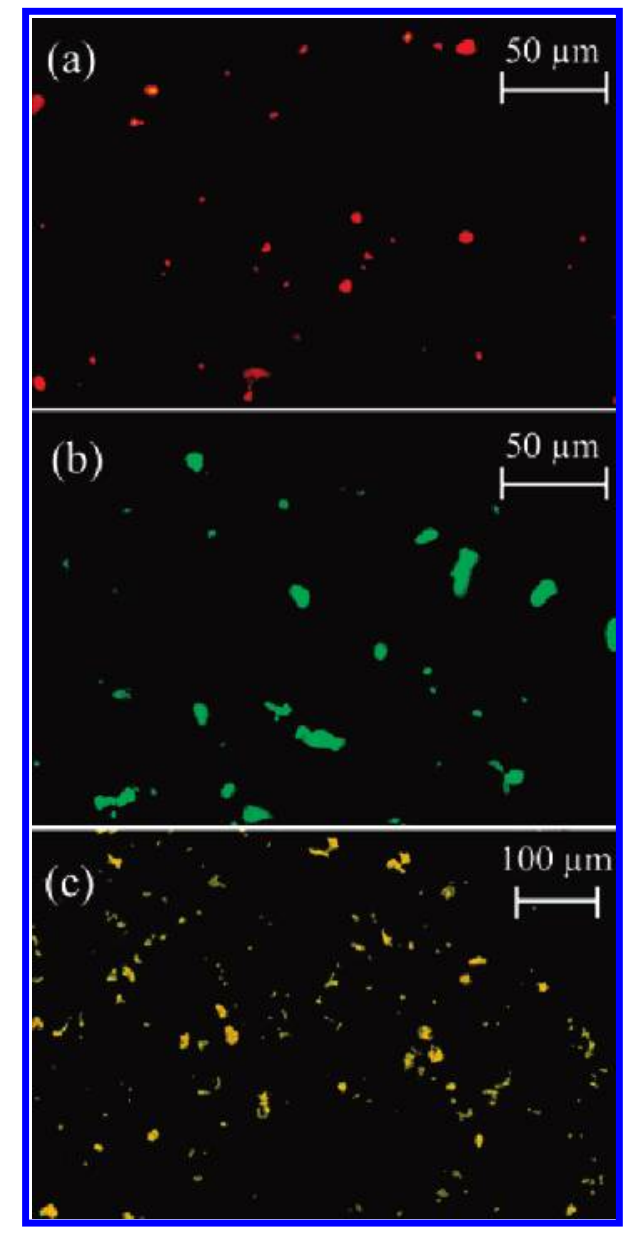

Figure 4. Photographic images (obtained with a fluorescence microscope) of the sensor materials: (a) Ru-dpp/ormosil (oxygen sensitive) beads in hydrogel; (b) HPTS/pHEMA ( $\mathrm{pH}$-sensitive) beads in hydrogel; (c) Ru-phen/PAN (temperature-sensitive) beads in polystyrene. Some particles are larger than the average size of the beads $(15 \mu \mathrm{m})$, which is due to particle aggregation.

The probes were chosen mainly by considering the criteria of (a) sensitivity (resolution over the range of interest); (b) photoexcitation with LEDs and-preferably-at one excitation wavelength only; (c) well-separated emissions or distinctly different decay times of luminescence; (d), readout via a commercial reader. The ranges of interest are as follows: $0-21 \mathrm{kPa}$ oxygen, $\mathrm{pH} 6-8$, $0-50{ }^{\circ} \mathrm{C}$. As a reader, we envisioned the use of two readers (Microx and pH-1 micro) rather than designing one more optoelectronic readout system. The Microx system has a 505-nm LED light source, the pH-1 Micro system a 470-nm LED. This also resulted in the design of a new sensing material for dual sensing of $\mathrm{DO} / \mathrm{pH}$ since the material that has been used earlier for planar sensing and imaging ${ }^{19}$ is not compatible with the $\mathrm{pH}-1$ microreader. Hence, the dye HPTS was used as a pH probe (as its absorbance matches the 470-nm LED of the reader) and covalently immobilized on poly(hydroxyethyl methacrylate) to avoid leaching out of the matrix.

In order to avoid a cross-interference of the temperature probe by oxygen in sensor SC-1, the indicator was immobilized into microbeads of poly (acrylonitrile), which is virtually gas-impermeable. The beads then were dispersed in the oxygen-permeable polystyrene matrix that contains the platinum porphyrin complex in dissolved form. The composite material SC-2 makes use of the two types of polymer microbeads-oxygen-sensitive and $\mathrm{pH}$ sensitive. For the preparation of the oxygen-sensitive microbeads, the oxygen indicator Ru-dpp was entrapped into highly gas permeable organically modified silica (ormosil). Thus, optimal sensitivity to oxygen was achieved. Microbeads containing HPTS immobilized on the amino-modified p-HEMA were used as $\mathrm{pH}$ probes. The Ru-dpp/ormosil and HPTS/pHEMA microbeads were dispersed in a biocompatible hydrogel matrix, which is permeable to both oxygen and protons. Platinum(II) porphyrin complexes as well as ruthenium(II) polypyridyl complexes are viable oxygen indicators, are widely used in optical sensing and imaging of oxygen, ${ }^{41-43}$ and are completely water-insoluble so that there is no risk of leaching.

The size of the beads is critical for sensor properties, such as sensitivity and dynamic response, and even small inhomogeneities can produce significant deviation in the case of microsensors. That is particularly true for the microsensor of type SC-2, since fluorescence intensity of the $\mathrm{pH}$ indicator is a referenced parameter. It should be mentioned that a microsensor of type SC-1 is less prone to such effects, because the decay time of both probes is measured independently. Figure 4 shows images of the microbeads obtained with fluorescence microscopy. Sensor cocktails containing only a single type of microbeads were coated on the polyester support and were allowed to dry to result in films. Typically, the size of the microbeads varies from 3 to $10 \mu \mathrm{m}$; in the case of HPTS/pHEMA and Ru-phen/PAN beads, some bigger aggregates (up to $15 \mu \mathrm{m}$ ) are also visible. As will be shown below, the beads are sufficiently small to obtain reproducible results.

Spectroscopy and Optoelectronic System. We envisioned using small optoelectronic readers rather than making use of bulky fluorometers or lock-in amplifiers. The readers used here (Microx and pH-1 Micro) are commercially available, comparably small (around $8 \times 5 \times 4 \mathrm{~cm}$ in size), and equipped with standard fiber-optic connectors (see Figure 2). In sensor type SC-1 $\left(\mathrm{O}_{2} /\right.$ $\mathrm{T})$, the two fluorescent probes are excited separately by two LEDs, and their emissions are spectrally isolated using two different filter sets. The temperature probe Ru-phen with its absorption peak at $448 \mathrm{~nm}$ readily absorbs light of the 470-nm blue LED. The oxygen probe $\mathrm{Ru}-\mathrm{TFPP}$ remains practically unexcited at this wavelength. Its excitation is performed at the so-called Q-band $\left(\lambda_{\max } 508 \mathrm{~nm}\right)$ using a 505-nm blue-green LED. The two emissions (with $\lambda_{\max }$ values of 585 and $650 \mathrm{~nm}$, respectively) are separated via two optical filters (see Figure 2). Moreover, a modulation frequency of $60 \mathrm{kHz}$ is applied in the temperature channel at which the luminescence of the oxygen probe is significantly demodulated (i.e., decreased in its relative contribution to the phase shift) since the optimal modulation frequency for Pt-TFPP is $5 \mathrm{kHz}$ only. If excited with the 505-nm LED, the ruthenium probe remains unexcited, while Pt-TFPP gives an oxygen-dependent phase shift.

In the case of the microsensor for $\mathrm{pH}$ and oxygen (SC-2; Figure 3), a modified dual lifetime referencing method (m-DLR) was applied that was described in some detail previously. ${ }^{44}$ The $\mathrm{pH}$ probe (HPTS) with its excitation/emission maximums of 468/

(41) Wolfbeis, O. S. L. Mater. Chem. 2005, 15, 2657-2669.

(42) Amao, Y. Microchim. Acta 2003, 143, 1-12.

(43) Wolfbeis, O. S.; Leiner, J. P.; Posch, H. E. Microchim. Acta 1986, 3, 359366

(44) Borisov, S. M.; Neurauter, G.; Schroeder, C.; Klimant, I.; Wolfbeis, O. S. Appl. Spectrosc. 2006, 60, 1167-1173.

Analytical Chemistry, Vol. 79, No. 22, November 15, 2007 


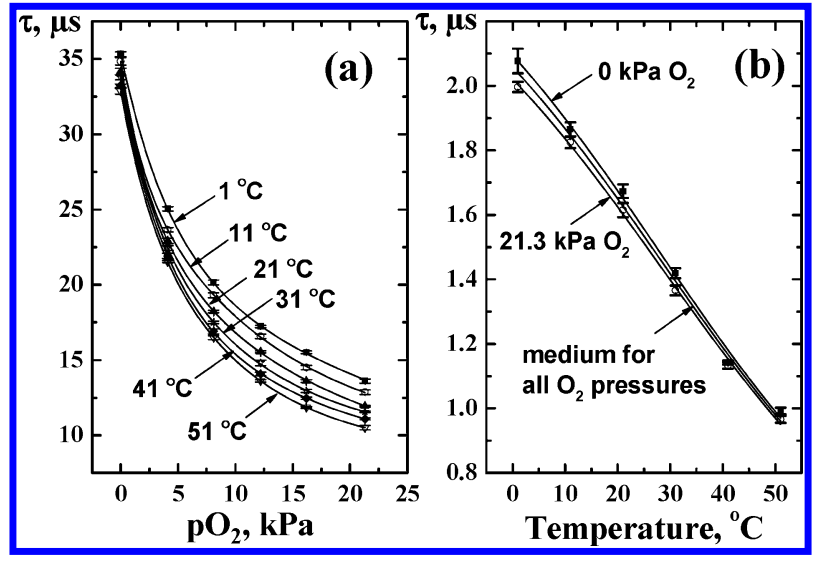

Figure 5. Response of the SC-1 microsensor (a) to dissolved oxygen and (b) to temperature. The larger standard deviation at low temperatures in (b) is due to the incomplete separation of the strong temperature signal from the weaker oxygen signal, and not due to quenching by oxygen.

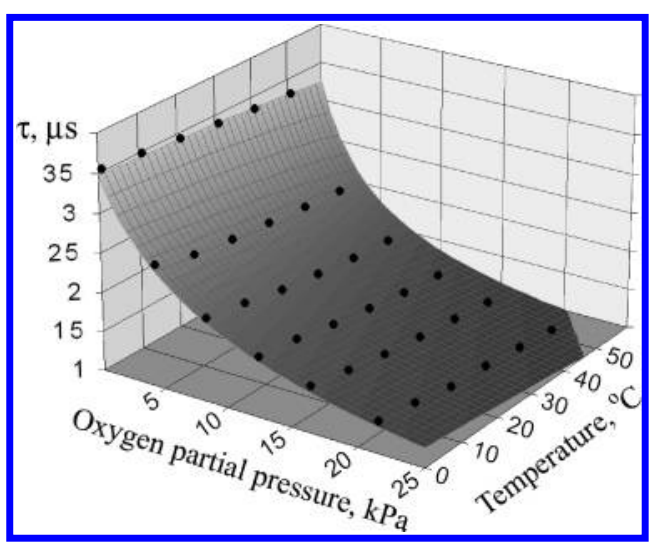

Figure 6. $3 \mathrm{D}$ plot of the response of the dual microsensor (using cocktail SC-1) to dissolved oxygen. Data points represent experimental data; the surface is the result of a mathematical fit.

$542 \mathrm{~nm}$, respectively, and the oxygen probe (Ru-dpp) with its absorption/emission maximums of 468/608 nm, respectively, were simultaneously excited with a single LED. The overall phase shift of the luminescences at above $550 \mathrm{~nm}$ is detected at modulation frequencies of 30 and $60 \mathrm{kHz}$, respectively. This results in information on the $\mathrm{pH}$-dependent fluorescence intensity of the $\mathrm{pH}$ probe (see below) and on the oxygen-dependent luminescence decay time of the oxygen probe. Because the fluorescence intensity is actually referenced against the decay time, such sensors do not suffer from drifts of the optoelectronic system and variations in the optical properties of the sensor material (including probe concentration and layer thickness) and of the sample (including turbidity, intrinsic sample coloration, and refractive index), which are common drawbacks of intensity-based sensors.

Response of the Sensor SC-1. The response of the microsensor based on SC-1 to dissolved oxygen and temperature is shown in Figure 5. PAN indeed very effectively shields the temperature indicator from quenching by oxygen, so that only a minor cross-sensitivity is observed (Figure 5b). For the sake of simplicity, we used a medium response curve obtained for all the microsensors at six oxygen partial pressures $(0,4.1,8.1,12.2,16.2$, and $21.3 \mathrm{kPa}$ ). The maximum error in temperature determination does not exceed $\pm 2{ }^{\circ} \mathrm{C}$ at the lowest temperature, and $\pm 0.5^{\circ} \mathrm{C}$ at the highest temperature.
The temperature dependence of the luminescence decay time can be described by an Arrhenius-type equation: ${ }^{18,45}$

$$
\tau=\left(k_{0}+k_{1} \exp \left(-\frac{\Delta E}{R(T+273)}\right)^{-1}\right.
$$

where $k_{0}$ is the temperature-independent decay rate for the excited-state deactivation, $k_{1}$ the pre-exponential factor, $\Delta E$ the energy gap between emitting level and higher excited-state level, $R$ the gas constant, and $T$ the temperature $\left(\right.$ in ${ }^{\circ} \mathrm{C}$ ). For the case of the medium response curve, $k_{0}$ was determined to be $4.0 \times$ $10^{5} \mathrm{~s}^{-1}, k_{1}$ was $2.81 \times 10^{10} \mathrm{~s}^{-1}$, and $\Delta E$ was $28.8 \mathrm{~kJ} \cdot \mathrm{mol}^{-1}$. The correlation coefficient $\left(r^{2}\right)$ was 0.998 . The function for temperature can be described by the following equation:

$$
T=-\frac{\Delta E}{\ln \left(\frac{\tau k_{0}-1}{\tau k_{1}}\right) R}-273
$$

As expected, the oxygen probe shows pronounced sensitivity to temperature. This is assumed to result from two factors, namely, (a) thermal quenching (which results in lower luminescence intensity and decay times at a given oxygen concentration at higher temperature); this can be clearly seen even in the absence of oxygen in Figure 5a; and (b) increasingly efficient quenching by oxygen at higher temperatures due to higher collisional rates.

Aside from the possibility of measurement of two parameters, the measurement of temperature also allows for the compensation of the two effects discussed above. This will be demonstrated later. Because the decay time of the oxygen probe is actually dependent on both oxygen partial pressure and temperature, the response can be best represented and fit as a 3D plot (Figure 6). The following empirical equation obtained (by the TableCurve 3D software) was found to adequately describe the $3 \mathrm{D}$ dependence $\left(r^{2}=0.998\right)$ :

$$
\tau=\left(a+b \mathrm{pO}_{2}+c T\right) /\left(1+d \mathrm{pO}_{2}+e T\right)
$$

where $\tau$ is the luminescence lifetime of the oxygen probe, $T$ is the temperature (in ${ }^{\circ} \mathrm{C}$ ), and $a, b, c, d$, and $e$, respectively, are empirical coefficients with values of $3.47 \times 10^{-5}, 5.59 \times 10^{-7},-2.81$ $\times 10^{-7}, 0.1163$, and $7.50 \times 10^{-3}$.

The calibration equation for oxygen can be expressed as follows:

$$
\mathrm{pO}_{2}=\frac{a-\tau-\tau e T+c T}{\tau d-b}
$$

Response of the Sensor SC-2. Figure 7 gives the experimental data. Measurement at two modulation frequencies (30 and $60 \mathrm{kHz}$ ) is adequate to end up with equations for the response of both analytes. The curves obtained with aqueous solutions of

(45) Liebsch, G.; Klimant, I.; Wolfbeis, O. S. Adv. Mater. 1999, 11, 1296-1299. 


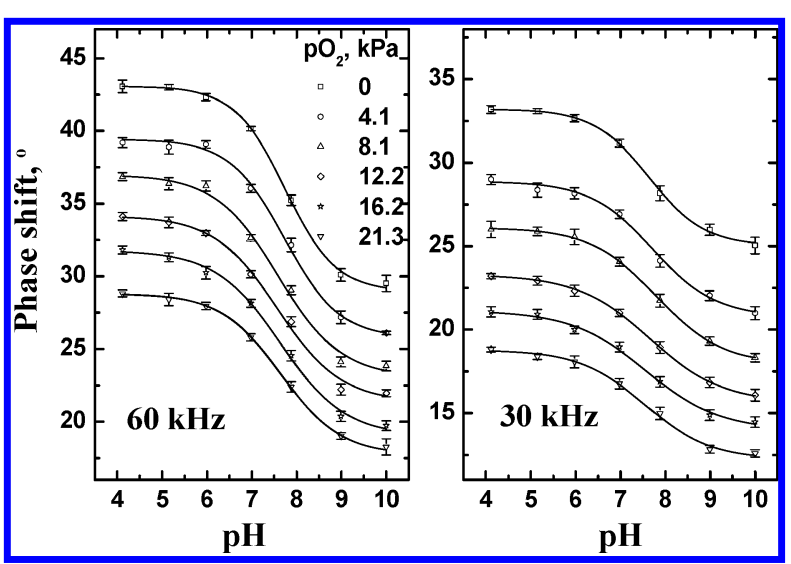

Figure 7. Response of the microsensor (using material SC-2) to $\mathrm{pH}$ at different oxygen partial pressures in buffered solutions $\left(\mathrm{pO}_{2}\right.$, $\mathrm{kPa}$ ). Lines represent a fit using eq 5 .
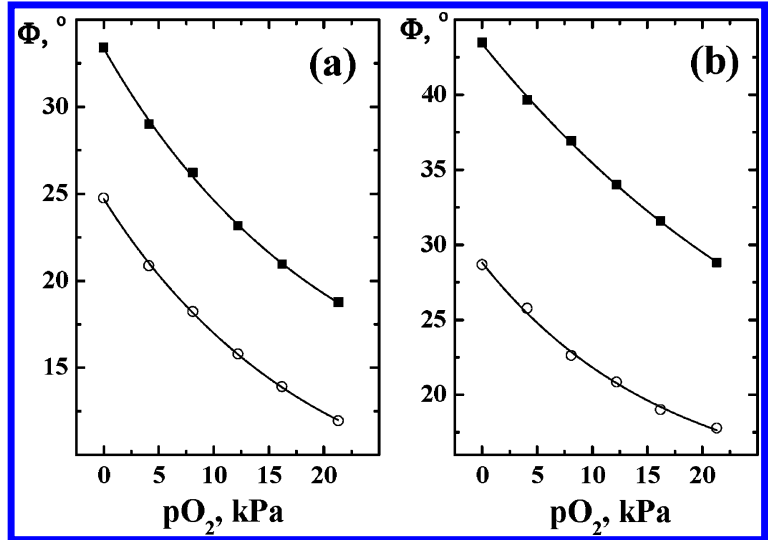

Figure 8. Dependence of the fitting parameters $A_{\max }$ and $A_{\min }$ on the oxygen partial pressure at modulation frequencies of $30 \mathrm{kHz}$ (a) and at $60 \mathrm{kHz}(\mathrm{b})$. Lines represent a fit using a monoexponential decay model.

various $\mathrm{pO}_{2}$ partial pressures can fit by the following equation $\left(r^{2}\right.$ $>0.997)$ :

$$
\Phi=\frac{A_{\max }-A_{\min }}{1+10^{\left(\mathrm{pH}-\mathrm{p} K_{a}\right) / x}}+A_{\min }
$$

where $\Phi$ is overall phase shift, $A_{\max }, A_{\min }$, and $x$ are numerical coefficients. $A_{\max }$ and $A_{\min }$ are numerical values of the phase shift of the $\mathrm{pH}$ probe in fully protonated $\left(A_{\min }\right)$ and fully deprotonated $\left(A_{\max }\right)$ form, respectively.

The $\mathrm{p} K_{\mathrm{a}}$ is an intrinsic property of the (immobilized) probe and found to be independent of the $\mathrm{pO}_{2}\left(\mathrm{p} K_{\mathrm{a}}=7.651 \pm 0.096\right.$ and $\mathrm{p} K_{\mathrm{a}}=7.651 \pm 0.07$; calculated for modulation frequencies of 30 and $60 \mathrm{kHz}$, respectively). The coefficient $x$ does not depend on $\mathrm{pO}_{2}$ either. It is slightly affected, however, by the modulation frequency used $(x=0.737 \pm 0.076$ and $x=0.663 \pm 0.060$ at modulation frequencies of 30 and $60 \mathrm{kHz}$, respectively). On the other side, coefficients $A_{\max }$ and $A_{\min }$ reflect the contribution of the oxygen probe (which is quenched) to overall phase shift. They are plotted versus $\mathrm{pO}_{2}$ in Figure 8.

The dependence of $A_{\min }$ and $A_{\max }$ on $\mathrm{pO}_{2}$ is best described by a monoexponential decay model of the form

$$
y=B \exp \left(-\mathrm{pO}_{2} / C\right)+D
$$

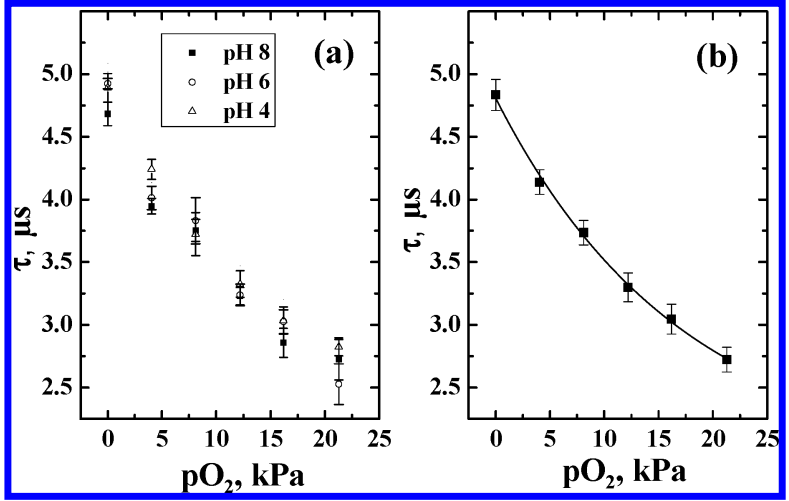

Figure 9. Response of the microsensor (type SC-2) to dissolved oxygen. (a) Response at different $\mathrm{pH}$ of solution; (b) medium curve, where points represent averaged values for all individual microsensors at all $\mathrm{pH}$ values; the curve represents a fit based on a monoexponential decay model.

Table 2. Numerical Data for the Fitting Parameters of Eq 6 at Various Modulation Frequencies modulation

frequency $(\mathrm{kHz})$

$A_{\max }=30$

$A_{\min }=30$

$A_{\max }=60$

$A_{\text {min }}=60$
$B$

22.52
18.77
31.68

\begin{tabular}{lc}
\multicolumn{1}{c}{$C$} & $D$ \\
20.40 & 10.83 \\
18.88 & 5.93 \\
34.60 & 11.72 \\
16.616 & 13.374 \\
\hline
\end{tabular}

where $y=A_{\max }$ or $A_{\min } ; B, C$, and $D$ are fitting parameters. The respective numerical values are given in Table 2. If $A_{\min }$ and $A_{\max }$ are introduced into the equation of the monoexponential decay model, the following equation for $\mathrm{pH}$ is obtained:

$$
\begin{aligned}
\mathrm{pH}= & 6.208+\ln \\
& \left(\frac{\left(B_{\min } \exp \left(-\mathrm{pO}_{2} / C_{\min }\right)+D_{\min }\right)-\Phi_{30 \mathrm{kHz}}}{\Phi_{30 \mathrm{kHz}}-\left(B_{\max } \exp \left(-\mathrm{pO}_{2} / C_{\max }\right)+D_{\max }\right)}\right) \cdot 0.8387
\end{aligned}
$$

Equation 7 can be applied for the calculation of $\mathrm{pH}$ both at 30 and $60 \mathrm{kHz}$.

The fluorescence intensity of the $\mathrm{pH}$ probe is referenced against the phase shift of the oxygen probe in the m-DLR method. As a result, the resulting "initial" (first approximation) $\mathrm{pH}$ value calculated from these data depends on the oxygen partial pressure, which therefore needs to be calculated first in order to compensate for its effect on the initial $\mathrm{pH}$ value.

The response function of the oxygen probe can be described by the formula given in eq 8 . If the frequencies $f_{1}$ and $f_{2}$ are smaller than $100 \mathrm{kHz}$, this equation eliminates the contributions of both the $\mathrm{pH}$ probe (HPTS) with its decay time in the order of $5 \mathrm{~ns}$ and, of course, also that of nanosecong-lived background fluorescence. Our working frequencies are 30 and $60 \mathrm{kHz}$. The overall phase shift is determined at two different modulation frequencies as

$$
\begin{array}{r}
\tau=\frac{1}{2 \pi}\left(f_{1}^{2}-f_{2}^{2} \pm\left(f_{2}^{2}-f_{1}^{2}-4\left(\cot \Phi_{2} f_{2} f_{1}^{2}-\cot \Phi_{1} f_{1} f_{2}^{2}\right)\right.\right. \\
\left.\left.\left(\cot \Phi_{2} f_{2}-\cot \Phi_{1} f_{1}\right)\right)^{1 / 2} / 2\left(\cot \Phi_{2} f_{2} f_{1}^{2}-\cot \Phi_{1} f_{1} f_{2}^{2}\right)\right)
\end{array}
$$

where $\Phi_{1}$ and $\Phi_{2}$ are the measured phase shifts at modulation frequencies $f_{1}$ and $f_{2}$. The response curves are shown in Figure 9 . 
For the sake of simplicity, only data for three different $\mathrm{pH}$ values are shown. It is evident that there is no cross-sensitivity to $\mathrm{pH}$.

Averaged values obtained for each individual microsensor at all pH's tested (i.e., pH 10, 9, 8, 7, 6, 5, and 4) were used to generate an average response curve (Figure 9b). Again, a fit employing a monoexponential decay model is adequate $\left(r^{2}\right.$ is $0.998)$. The fit parameters $B, C$, and $D$ are $2.864 \times 10^{-6}, 16.585$,and $1.942 \times 10^{-6}$, respectively. Hence, the equation for calculating the $\mathrm{pO}_{2}$ can be written as follows:

$$
\mathrm{pO}_{2}=-16.585 \ln \left(\frac{\tau-1.942 \times 10^{-6}}{2.864 \times 10^{-6}}\right)
$$

where $\tau$ is the decay time calculated via eq 8 .

Response Times. The response of microsensor SC- 1 to temperature is instantaneous and limited obviously by the rates of heat transfer. Indeed, the phase shift becomes constant within $1 \mathrm{~s}$ after placing the tip of the fiber in a solution of different temperature. The situation was different for oxygen. When cycling the $\mathrm{pO}_{2}$ of the solution from 0 to $21.3 \mathrm{kPa}$ and back, the time needed for the $95 \%$ of the total signal change to occur $\left(t_{95}\right)$ was similar for both directions and totaled $70 \mathrm{~s}$ on average. The response to oxygen of microsensor $\mathrm{SC}-2\left(\mathrm{pH} / \mathrm{O}_{2}\right)$ took $30 \mathrm{~s}$ on average in both directions. The response of the $\mathrm{pH} / \mathrm{O}_{2}$ sensor to $\mathrm{pH}\left(t_{95}\right)$ varied from batch to batch but averaged 2 min when going from $\mathrm{pH} 4$ to $\mathrm{pH} \mathrm{9,} \mathrm{and} 3 \mathrm{~min}$ in the reverse direction. This is rather long and may be explained by a diffusional barrier in the polymers and, possibly, electrostatic repulsion of protons in the $\mathrm{pH}$ beads.

Photostability. Both types of dual microsensors make use of highly photostable indicators, specifically Pt-TFPP (for $\mathrm{O}_{2}$ ), Ruphen (T), Ru-dpp $\left(\mathrm{O}_{2}\right)$, and HPTS $(\mathrm{pH})$. Since the light intensities used to induce their luminescence at the tip of the optical fibers significantly exceed those used in case of planar sensor foils, photodegradation can be a critical issue even for indicators that possess good photostability in other conditions. Nevertheless, we have found that the microsensors of types SC- 1 and SC-2 exhibited surprisingly good photostability. In fact, in the case of $\mathrm{O}_{2} / \mathrm{T}$, no noticeable drift in phase shift and luminescence intensity was observed after 100 data acquisitions at oxygen-free solutions, and 500 data acquisitions at air-saturated solutions at $25^{\circ} \mathrm{C}$. Similarly, the phase shift of the temperature indicator (excitated with the 470-nm LED) was unaltered after having acquired 1000 data points at different temperatures.

The $\mathrm{pH} / \mathrm{O}_{2}$ microsensor displayed no drift at $\mathrm{pH} 4$ under 0 and $21.3 \mathrm{kPa} \mathrm{O}_{2}$ (the $\mathrm{pH}$ indicator is not photoexcited by the green LED) when operated over $2 \mathrm{~h}$ (equivalent to 740 data points). Also, no drift (phase shift) was observed for the microsensor at $\mathrm{pH} 9$ and oxygen-free condition. However, an increase in the overall phase shift of $\sim 0.35^{\circ}$ (angle degree) per 500 data acquisition points was observed at $\mathrm{pH} 9$ under air saturation, which was accompanied by a decrease of luminescence intensity ( 3\% per 500 measuring points). Assuming the acquisition of one data point per $10 \mathrm{~s}$ (as is usual in practice), a drift of $0.35^{\circ}$ (equivalent to $0.1-0.2 \mathrm{pH}$ units depending on actual $\mathrm{pH}$ ) will be found after an operation time of $8.3 \mathrm{~h}$. The total phase shift at $60 \mathrm{kHz}$ on going from $\mathrm{pH} 5$ to $\mathrm{pH} 10$ is typically $10-13^{\circ}$ (depending on $\mathrm{pO}_{2}$ ). The increase in

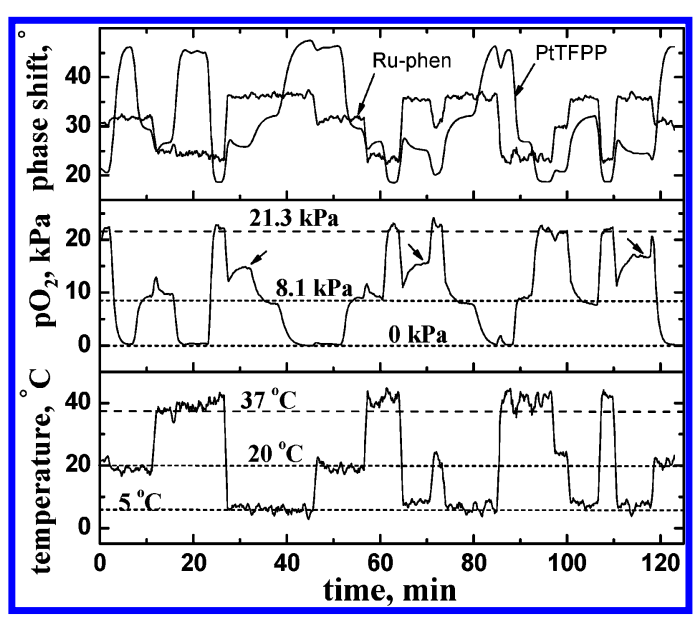

Figure 10. Performance of the dual microsensor for $\mathrm{pO}_{2}$ and temperature. Experimental phase shifts were measured at 470 - and 505-nm excitation. Oxygen partial pressures and temperatures were adjusted as given in the Experimental Section.

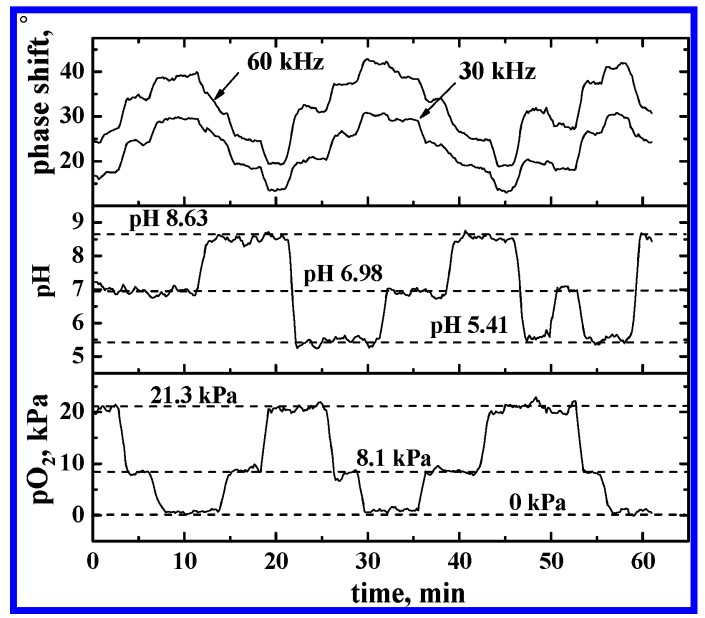

Figure 11. Performance of the SC-2 microsensor. Experimental phase shifts were measured at $470-\mathrm{nm}$ excitation at modulation frequencies of 30 and $60 \mathrm{kHz}$. The $\mathrm{pH}$ values and oxygen partial pressures were calculated via eqs 7 and 9 , respectively. All measurements were performed at $25{ }^{\circ} \mathrm{C}$ in aqueous solutions of defined $\mathrm{pH}$ $(8.63,6.98,5.41)$ as measured with a pH electrode or in plain water of a defined $\mathrm{pO}_{2}$ that was adjusted with a gas blender.

phase shift indicates decomposition of the $\mathrm{pH}$ indicator and is likely to be due to singlet oxygen generation by the oxygensensitive probe in the presence of molecular oxygen.

Validation. The performance of the microsensors was continuously tested at various conditions of temperature and $\mathrm{pO}_{2}$ (for $\mathrm{SC}-1$ microsensor) and of $\mathrm{pH}$ and $\mathrm{pO}_{2}$ (for $\mathrm{SC}-2$ ). The experimentally determined phase shifts and the data calculated for $\mathrm{pO}_{2}$, temperature, and $\mathrm{pH}$ are shown in Figures 10 and 11. For the SC-1 microsensor, temperature was calculated first using eq 2; theses values were then introduced into eq 4 to calculate oxygen partial pressure. In the case of the SC-2 microsensor, eq 8 was used to determine $\mathrm{pO}_{2}$, and $\mathrm{pH}$ values were then calculated with the help of eq 6 . As can be seen from Figure 10, microsensors are indeed capable of providing reliable and continuous information on oxygen partial pressure and temperature.

The lowest precision for temperature measurements is observed at above $45{ }^{\circ} \mathrm{C}$ (where deviations up to $4{ }^{\circ} \mathrm{C}$ were observed). This can be explained by the lower luminescence 
intensity of the temperature probe at higher temperatures and, thus, a lower signal-to-noise ratio. If a better precision is needed, the ratio of the temperature-sensitive beads to the oxygen-sensitive beads in the sensor chemistry (SC-1) needs to be increased, albeit possibly at the expense of the precision of oxygen measurements at room temperature. In any case, the results given in Figure 10 show that temperature-compensated oxygen sensing gives data that are very close to the respective parameters adjusted. However, the $\mathrm{pO}_{2}$ values calculated for air-saturated solutions $(21.3 \mathrm{kPa})$ at $5{ }^{\circ} \mathrm{C}$ (indicated in the figure with two arrows) initially are lower than expected but slowly increase within a few minutes. We assume that this is caused by an experimental artifact since the solution was cooled to $5^{\circ} \mathrm{C}$ but not agitated.

An experiment similar to the one described for the $\mathrm{O}_{2} / \mathrm{T}$ sensor (Figure 10) was performed with the $\mathrm{pH} / \mathrm{O}_{2}$ dual microsensor. As

(46) Moreno-Bondi M. C.; Wolfbeis, O. S.; Leiner M. J. P.; Schaffar, B. P. H.; Anal. Chem. 1990, 62, 2377-2380. can be seen from Figure 11, continuous dual sensing is possible in a highly reproducible fashion over the whole period of the experiment, and the sensor responds fully reversibly to the changes of $\mathrm{pH}$ and oxygen partial pressure. We now envision the design of triple sensors.

The microsensor for $\mathrm{pH} / \mathrm{O}_{2}$ was also tested in a Luria broth medium. As long as solutions did not contain any fluorophores, the results in essence parallel those obtained with test solutions. The sensor is, however, affected by the presence of intrinsic fluorophores in the sample solution which cause background fluorescence. In this case, optical isolation of the sensor tip with either white particles (such as $\mathrm{TiO}_{2}{ }^{18}$ ) or with a black coating (such as a mixture of polymer D4 and carbon black ${ }^{39,46}$ ) is required.

Received for review March 13, 2007. Accepted September 13, 2007.

$\mathrm{AC} 070514 \mathrm{H}$ 